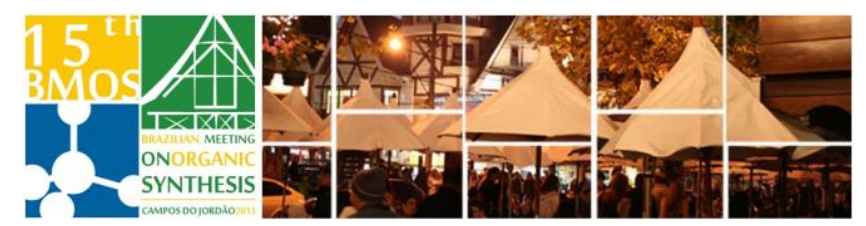

\title{
Synthesis of new heterocyclic isosteres of L-buthionine sulfoximine (L-BSO)
}

\author{
Thomas P.; Armijo R.; Tapia R. A.; Salas C. \\ Facultad de Química Orgánica, Pontificia Universidad Catolica de Chile \\ Vicuña Mackenna 4860, Macul, 7820436, Santiago, Chile \\ *E-mail:cosalas@uc.cl
}

Keywords: L-Butionine sulfoximine, isosters, heterocycles.

\section{INTRODUCTION}

Glutathione (GSH) in Trypanosoma cruzi, is a pivotal precursor of trypanothione, $\mathrm{T}(\mathrm{SH})_{2}$, the main molecule that protects this parasite of the toxic effects produced by certain drugs, hydrogen peroxide and free radicals ${ }^{1}$. In GSH synthesis, Glutamate Cysteine Ligase (GCL) plays a fundamental role because it catalyzes the limiting step and is inhibited by L-Buthionine Sulfoximine 1 $(\mathrm{L}-\mathrm{BSO})^{2}$. Recent studies have shown that the reduction in the synthesis of GSH by L-BSO increase the trypanocidal effect of nifurtimox and benznidazol in in vitro models of Chagas's diseases $^{3}$. In this context, we were interested in developing new heterocyclic isosters of L-BSO. With this purpose we first carried out a 3D model of GCL of $T$. cruzi, to design new derivatives of L-BSO ${ }^{4}$.

\section{RESULTS AND DISCUSSION}

Using the information provided by the 3D model, we modified the zwitterionic part of L-BSO with different heterocyclic rings. This new analogs may act as heterocyclic isosters of BSO with improved biodisponibility (Fig.1).

Figure 1. Chemical structure of L-BSO and heterocyclic isosters.

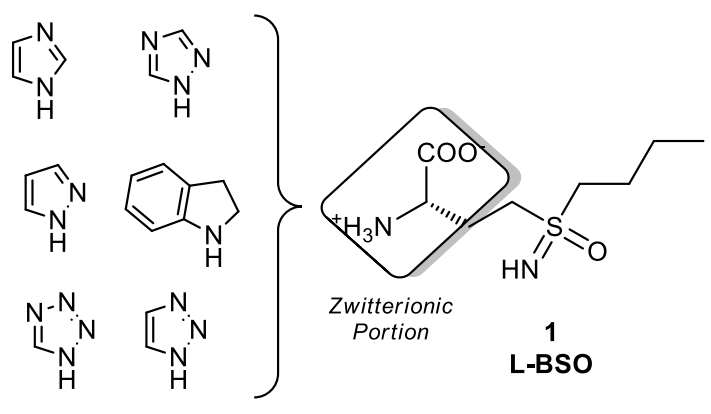

Heterocyclic isosters

With this information in hand, a synthetic pathway to obtain these compounds is based on the retrosynthetic scheme shown in figure 2 .
Figure 2. Retrosynthetic analysis for obtain isosters of $L$ BSO.

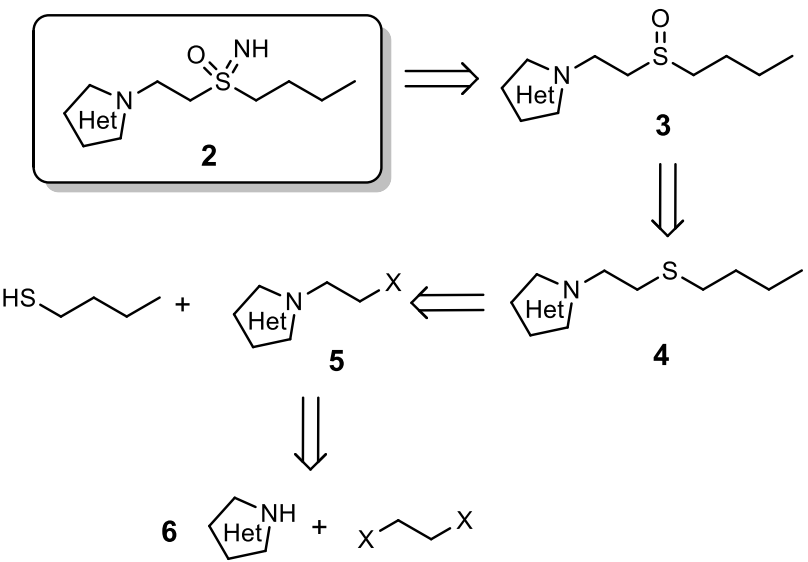

Alkylation of selected heterocyclics (6) with 1,2-dihaloethane followed by $S_{N} 2$ type reaction of $\mathbf{5}$ with 1-buthanetiol gave sulfide intermediates 4 . These sulfides were oxidized with $m$ chloroperbenzoic acid (MCPBA) under usual conditions, to obtain sulfoxides 3 in good yields (57$78 \%$ ). Finally, sulfoxides $\mathbf{3}$, were treated with o-mesitylenesulfonylhydroxylamine $(\mathrm{MSH})$ to afford sulfoximines 2 (34-75\%).

\section{CONCLUSION}

We have obtained a series of new heterocyclic isosters of L-BSO, which are under study to evaluate their trypanocidal effects.

\section{ACKNOWLEDGEMENTS}

We thank FONDECYT project № 11085027 , CONICYT for grant № 21100843 and Becas Chile for grant № 24121409 .

\section{REFERENCES}

1 Joo, Y.; Whee, J.; Hee, E.; Kyung, H.; Wong, H.; Kiem, Y. Free Radical Biol. \& Med.2004, 37, 272.

${ }^{2}$ Hibi, T.; Nii, H.; Nakatsu, T.; Kimura, A.; Kato, H.; Hiratake, J. Prod. Nat. Ac. Sci. 2004, 101, 15052.

3 Faundez, M.; Pino, L.; Letelier, P.; Ortiz, C.; Lopez, R.; Seguel, C. Ferreira, J.; Pavani, M.; Morello, A.; Maya, J. Antimicrob. Che. 2005, 49, 126.

${ }^{4}$ Lagos, C.; Araya-Secchi, R.; Thomas, P.; Pérez-Acle, T.; Tapia, R. A.; Salas, C. J. Mol.Model. 2012, 18, 2055. 